\title{
Higher topological complexity and its symmetrization
}

\author{
IBAI BASABE \\ JESÚS GONZÁLEZ \\ YULI B RUDYAK \\ DAI TAMAKI
}

\begin{abstract}
We develop the properties of the $n^{\text {th }}$ sequential topological complexity $\mathrm{TC}_{n}$, a homotopy invariant introduced by the third author as an extension of Farber's topological model for studying the complexity of motion planning algorithms in robotics. We exhibit close connections of $\mathrm{TC}_{n}(X)$ to the Lusternik-Schnirelmann category of cartesian powers of $X$, to the cup length of the diagonal embedding $X \hookrightarrow X^{n}$, and to the ratio between homotopy dimension and connectivity of $X$. We fully compute the numerical value of $\mathrm{TC}_{n}$ for products of spheres, closed 1-connected symplectic manifolds and quaternionic projective spaces. Our study includes two symmetrized versions of $\mathrm{TC}_{n}(X)$. The first one, unlike Farber and Grant's symmetric topological complexity, turns out to be a homotopy invariant of $X$; the second one is closely tied to the homotopical properties of the configuration space of cardinality- $n$ subsets of $X$. Special attention is given to the case of spheres.
\end{abstract}

55M30; 55R80

\section{Introduction, main results and organization}

A motion planning algorithm (mpa) for an autonomous system (robot) $S$ is a rule assigning to each pair $(A, B)$ of initial-final positions of $S$ a (continuous) motion from $A$ to $B$; see Latombe [20] and LaValle [21]. If $X$ stands for the space of all possible states of $S$, and $P(X)$ is the space of all paths $\gamma:[0,1] \rightarrow X$, then an mpa for $S$ is a (nonnecessarily continuous) section for the endpoints evaluation map $e: P(X) \rightarrow X \times X$ defined as $e(\gamma)=(\gamma(0), \gamma(1))$.

For practical applications one is interested in continuous mpa's. However it is easy to see that the endpoints evaluation map $e$ admits a continuous section if and only if the space of states $X$ is contractible. An alternative to continuity is to look at the Švarc genus of the map $e$, which leads to Farber's concept of topological complexity. This gives a way of recognizing mpa's with the least possible order of instability; see [6, Section 4]. The recognition is done directly from the homotopical properties of the space of states of the robot. 
Definition (Farber) Given a path-connected topological space $X$, the topological complexity of $X, \mathrm{TC}(X)$, is the least positive integer $k$ such that the cartesian product $X \times X$ can be covered by $k$ open subsets $U_{1}, U_{2}, \ldots, U_{k}$ on each of which $e$ admits a continuous section $s_{i}: U_{i} \rightarrow P(X)$. Each pair $\left(U_{i}, s_{i}\right)$ is called a local motion planner with domain $U_{i}$. We set $\operatorname{TC}(X)=\infty$ if no such $k$ exists.

A symmetrized version of topological complexity arises when attention is restricted to local planners for which the motion from $A$ to $B$ is the reverse of the motion from $B$ to $A$; see Farber and Grant [9]. A number of properties of topological complexity and symmetric topological complexity were found by Farber in $[5 ; 7 ; 8]$, Farber and Grant in [9; 10] and Farber and Yuzvinsky in [12]. The papers by Farber, Tabachnikov and Yuzvinsky [11] and the second author and Landweber [15] identify these concepts in the case of real projective spaces as their immersion and embedding dimensions, respectively.

This paper is concerned with the third author's generalization of the above concepts. In such a view, the motion planning does not only depend on a couple of initial-final states of a robot, but in a sequence of prescribed intermediate stages that the robot should reach through the motion. Such a setting is standard in industrial production processes in which the manufacture of a given good goes through a series of production steps. The corresponding need to identify best possible sequential motion planning algorithms leads to a homotopy invariant $\mathrm{TC}_{n}(X)$, the $n^{\text {th }}$ topological complexity of $X$, introduced by the third author in [23] and reviewed in Section 2 (where we use normalized notation, ie in such a way that contractible spaces have $\mathrm{TC}_{n}=0$ ).

In Section 3 we discuss basic properties of $\mathrm{TC}_{n}$, including methods for calculating this homotopy invariant. In Theorem 3.9 we describe optimal bounds for $\mathrm{TC}_{n}(X)$ : lower bounds are given in terms of the cup length of elements in the kernel of the iterated diagonal, whereas connectivity and homotopy dimension of $X$ lead to upper bounds. The subadditivity of $\mathrm{TC}_{n}$ is settled in Proposition 3.11. As an application, we obtain the full determination of the numerical value of $\operatorname{TC}_{n}(X)$ when $X$ is either a product of spheres (Corollary 3.12), a closed simply connected symplectic manifold (Corollary 3.15) or a quaternionic projective space (Corollary 3.16).

Many of our results generalize existing properties of Farber's TC. For instance, in Corollary 3.3 we show the following close connection between higher topological complexity and the Lusternik-Schnirelmann category of cartesian powers of spaces.

Theorem For a path-connected space $X, \operatorname{cat}\left(X^{n-1}\right) \leq \operatorname{TC}_{n}(X) \leq \operatorname{cat}\left(X^{n}\right)$.

Theorem 3.5 below gives $\operatorname{TC}_{n}(G)=\operatorname{cat}\left(G^{n-1}\right)$ for a path-connected topological group $G$, which extends the $n=2$ property proved by Farber in [6, Lemma 8.2]. 
Lupton and Scherer have recently proved that this property extends to not necessarily homotopy-associative Hopf spaces; see Lupton and Scherer [22].

Section 4 deals with symmetric versions of higher topological complexity. We begin by introducing $\mathrm{TC}^{\Sigma}(X)$, a variation of the symmetric topological complexity $\operatorname{TC}^{S}(X)$ introduced in [9]. We prove that the numerical values of the two invariants differ at most by a unit (Proposition 4.2). Such a fact should be prised by noticing that, although Farber and Grant observe that $\mathrm{TC}^{S}(X)$ is not a homotopy invariant, $\mathrm{TC}^{\Sigma}(X)$ depends only on the homotopy type of $X$. It should be noted that the homotopy invariance also fails in general for the monoidal topological complexity introduced by Iwase and Sakai (see [16, Definition 1.3 and Remark 1.4]), where the stasis property is imposed on the motion planning problem, instead of the symmetry condition we impose on $\mathrm{TC}^{\Sigma}$. We construct the corresponding higher analogues $\mathrm{TC}_{n}^{S}$ and $\mathrm{TC}_{n}^{\Sigma}$, and prove the homotopy invariance of the latter (Proposition 4.7).

The calculation of $\mathrm{TC}_{n}^{S}$ can turn out to be an extremely difficult task, mainly due to what seems to be the limited current knowledge of precise homotopy information about braid spaces (even braid manifolds, for that matter). In Section 5 we exhibit evidence leading to the conjecture that

$$
\mathrm{TC}_{n}^{S}\left(S^{k}\right) \leq[(n+2)(k-1)+4](n-1) / 2 k
$$

holds for integers $k \geq 1$ and $n \geq 2$. In particular, we observe in Corollary 5.5 that the equality $\mathrm{TC}_{n}^{S}\left(S^{k}\right)=2(n-1)$ holds provided $n=2$ or $k=1$.

Acknowledgements The first author wishes to thank Michael Farber, Jesús González, Dirk Schütz and the Mathematisches Forschungsinstitut Oberwolfach for organizing a wonderful Arbeitsgemeinschaft mit aktuellem Thema in Topological Robotics. The third author is grateful for the support during a visit at the Max Planck Institute for Mathematics in Bonn, Germany. The fourth author would like to thank the Centro di Ricerca Matematica Ennio De Giorgi, Scuola Normale Superiore di Pisa, for supporting his participation in the research program "Configuration Spaces: Geometry, Combinatorics and Topology," during which a part of his work on this paper was done. The second, third and fourth authors were partially supported, respectively, by Conacyt Research Grant 221221, Simons Foundation Grant 209424 and Grants-in-Aid for Scientific Research, Ministry of Education, Culture, Sports, Science and Technology, Japan, 23540082. The authors wish to express their most sincere gratitude to Peter Landweber for valuable suggestions on earlier versions of this paper, and for pointing out an important extension of the authors' original evidence for the conjectural assertion in (13). 


\section{Preliminaries on notation}

We use the normalized version of Švarc's concept of the genus of a map [24].

Definition 2.1 The $\breve{S}$ varc genus (also known as sectional category) of a map $p: E \rightarrow B$ is the least number $k$ such that there is an open covering $U_{0}, U_{1}, \ldots, U_{k}$ of $B$ for which the restriction of $p$ to each $U_{i}(i=0,1, \ldots, k)$ admits a homotopy section, ie a (continuous) map $s_{i}$ : $U_{i} \rightarrow E$ such that $p s_{i}$ is homotopic to the inclusion $U_{i} \hookrightarrow B$. We agree to set $\mathfrak{g e n u s}(f)=-1$ for $f: X \rightarrow Y$ with $X=\varnothing=Y$.

The following result, proved in [24, Proposition 22, page 84] (see also the comments in [24, Section 1, page 54]), will be used in the proof of Proposition 3.11. Here we agree that a normal space is, by definition, required to be Hausdorff. This convention will also be in force throughout Section 3.

Proposition 2.2 Let $f \times f^{\prime}: X \times X^{\prime} \rightarrow Y \times Y^{\prime}$ be the product of two maps $f: X \rightarrow Y$ and $f^{\prime}: X^{\prime} \rightarrow Y^{\prime}$. If $Y \times Y^{\prime}$ is normal, then $\mathfrak{g e n u s}\left(f \times f^{\prime}\right) \leq \mathfrak{g e n u s}(f)+\mathfrak{g e n u s}\left(f^{\prime}\right)$.

Definition 2.3 Let $X$ be a path-connected space. The $n^{\text {th }}$ topological complexity of $X, \mathrm{TC}_{n}(X)$, is the Švarc genus of the fibration

$$
e_{n}^{X}=e_{n}: X^{J_{n}} \rightarrow X^{n}, \quad e_{n}(\gamma)=\left(\gamma\left(1_{1}\right), \ldots, \gamma\left(1_{n}\right)\right),
$$

where $J_{n}$ is the wedge of $n$ closed intervals $[0,1]$ (each with $0 \in[0,1]$ as the base point), and $1_{i}$ stands for 1 in the $i^{\text {th }}$ interval.

We note that (2) is the standard fibrational substitute for the iterated diagonal map $d_{n}=d_{n}^{X}: X \rightarrow X^{n}$, so $\operatorname{TC}_{n}(X)=\mathfrak{g e n u s}\left(d_{n}^{X}\right)$. More generally, for a contractible space $Y_{n}$ with $n$ distinct distinguished points $v_{1}, \ldots, v_{n} \in Y_{n}$, consider the evaluation map $e_{Y_{n}}: X^{Y_{n}} \rightarrow X^{n}, e_{Y_{n}}(f)=\left(f\left(y_{1}\right), \ldots, f\left(y_{n}\right)\right)$. Because of the contractibility of $Y_{n}$, the genus of $e_{Y_{n}}$ is equal to $\mathrm{TC}_{n}(X)$; the proof is just as the one in [23, Remark 3.2.5]. In particular, we can take $Y_{n}$ to be a tree with $n$ leaves, or the unit interval $I_{n}$, say with distinguished points $v_{i}=(i-1) /(n-1), i=1, \ldots, n$. In the latter case we see that the $n^{\text {th }}$ higher topological complexity gives a topological measure of the complexity of the motion planning problem where the robot is required to visit $n$ ordered prescribed stages. For this reason, we also refer to $\mathrm{TC}_{n}$ as the $n^{\text {th }}$ sequential topological complexity. Farber's $\mathrm{TC}$ is $\mathrm{TC}_{2}+1$.

Other fibrations (which not necessarily give fibrational substitutes of the iterated diagonal) can be used to define $\mathrm{TC}_{n}$. Indeed, let $G_{n}$ be any connected graph where $n$ ordered distinct vertices $v_{1}, \ldots, v_{n}$ have been selected. We assert that the evaluation 
map $e_{G_{n}}: X^{G_{n}} \rightarrow X^{n}$ at the chosen vertices has $\mathfrak{g e n u s}\left(e_{G_{n}}\right)=\mathrm{TC}_{n}(X)$. To see this, choose maps $I_{n} \rightarrow G_{n} \rightarrow J_{n}$ preserving the selected vertices. For instance, the latter map can be taken so to collapse most of $G_{n}$ to the base point in $J_{n}$, except that the first half of each directed edge $\left(v_{i}, v\right)$ in $G_{n}$ is mapped linearly onto the directed edge $\left(1_{i}, 0\right)$ in $J_{n}$ (in particular vertices $v_{i}$ are mapped to vertices $1_{i}$ ). Since the induced maps $X^{J_{n}} \rightarrow X^{G_{n}} \rightarrow X^{I_{n}}$ are compatible with the three evaluation maps, we get $\mathfrak{g e n u s}\left(e_{I_{n}}\right) \leq \mathfrak{g e n u s}\left(e_{G_{n}}\right) \leq \mathfrak{g e n u s}\left(e_{J_{n}}\right)$. But, as explained in the paragraph above, the extremes in the previous chain of inequalities agree with $\mathrm{TC}_{n}(X)$.

We close this section setting notation relevant to the construction (in Section 5) of our two symmetric versions of higher topological complexity.

The (left) action of the symmetric group $\Sigma_{n}$ on $\left\{1_{1}, \ldots, 1_{n}\right\}$ extends to one on $J_{n}$. This yields corresponding (right) $\Sigma_{n}$-actions on $X^{n}$ and $X^{J_{n}}$ in such a way that (2)

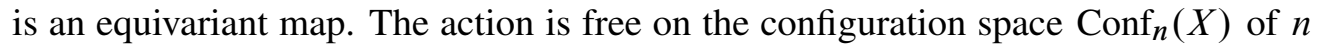
ordered distinct points in $X$ and, consequently, on $e_{n}^{-1}\left(\operatorname{Conf}_{n}(X)\right)$. Thus, at the level of orbit spaces we get a fibration

$$
\varepsilon_{n}^{X}=\varepsilon_{n}: Y_{n}(X) \rightarrow \operatorname{Braid}_{n}(X),
$$

where $Y_{n}(X)=e_{n}^{-1}\left(\operatorname{Conf}_{n}(X)\right) / \Sigma_{n}$ and $\operatorname{Braid}_{n}(X)=\operatorname{Conf}_{n}(X) / \Sigma_{n}$, the latter being the usual "braid" configuration space of cardinality- $n$ subsets of $X$.

We think of $\mathfrak{g e n u s}\left(\varepsilon_{n}^{X}\right)$ as giving a measure for the topological complexity of the $n^{\text {th }}$ ubiquitous motion planning problem on $X$. This concept serves in Section 4 as the building block relating our two symmetrized forms of $\mathrm{TC}_{n}$; see Theorem 4.8 and Definition 4.13. Section 5 will be devoted to exploring $\mathfrak{g e n u s}\left(\varepsilon_{n}^{S^{k}}\right)$.

Note that the commutative diagram (where horizontal arrows are canonical projections)

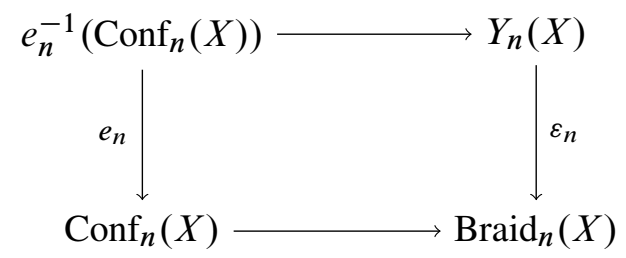

is a pullback square, so that (local) sections of $\varepsilon_{n}$ correspond to $\Sigma_{n}$-equivariant (local) sections of $e_{n}$. In particular, the homotopy fiber of $\varepsilon_{n}$ is $(\Omega X)^{n-1}$, just as for $e_{n}$; see [23, Remark 3.2.3]. For instance, a copy of $(\Omega X)^{n-1}$ sits inside the fiber of $e_{n}$ over an $n$-tuple $\left(x_{1}, x_{2}, \ldots, x_{n}\right)$ as the strong deformation retract consisting of multipaths $\left\{\gamma_{j}\right\}_{j=1}^{n}$ for which $\gamma_{1}$ is the constant path at $x_{1}$. Here and below, the term "multipath" refers to an element $\gamma \in X^{J_{n}}$, and we will use the notation $\gamma=\left\{\gamma_{j}\right\}_{j=1}^{n}$, where $\gamma_{j}$ is the restriction of $\gamma$ to the $j^{\text {th }}$ wedge summand of $J_{n}$. 


\section{Properties of higher topological complexity}

The higher topological complexities of a space $X$ are closely related to the category of cartesian powers of $X$. The first indication of such a property comes from the inequality

$$
\mathrm{TC}_{n}(X) \leq \operatorname{cat}\left(X^{n}\right)
$$

which is an immediate consequence of the well known fact that the Švarc genus of a fibration does not exceed the category of the base space. On the other hand, the inequality $\operatorname{cat}(X) \leq \mathrm{TC}_{2}(X)$ is well known, and can be generalized to the following.

Proposition 3.1 For any path-connected space $X$,

$$
\operatorname{cat}\left(X^{n-1}\right) \leq \mathrm{TC}_{n}(X) .
$$

Proof Let $\mathrm{TC}_{n}(X)=k$ and choose a covering $B_{0} \cup B_{1} \cup \cdots \cup B_{k}=X^{n}$ such that there is a continuous section $s_{i}$ for $e_{n}^{X}$ over $B_{i}$ for $i=0, \ldots, k$. Let $p: X^{n} \rightarrow X$ be the projection onto the first factor, choose $x_{1} \in X$, and put $A_{i}=p^{-1}\left(x_{1}\right) \cap B_{i}$. Note that $\left\{A_{i}\right\}_{i=0}^{k}$ is an open cover for $p^{-1}\left(x_{1}\right)$. Since $p^{-1}\left(x_{1}\right)$ is homeomorphic to $X^{n-1}$, it suffices to show that each $A_{i}$ is contractible within $p^{-1}\left(x_{1}\right)$.

For a point $\left(x_{1}, x_{2}, \ldots, x_{n}\right) \in A_{i}$ consider the $n$ paths $\gamma_{1}, \ldots, \gamma_{n}$ making up the multipath $s_{i}\left(x_{1}, x_{2}, \ldots, x_{n}\right)=\left\{\gamma_{j}\right\}_{j=1}^{n}$. Then $\gamma_{j}(1)=x_{j}$ and $\gamma_{j}(0)=x_{0}$ for some $x_{0} \in X$ which is independent of $j \in\{1, \ldots, n\}$. Then, the constant path $\delta_{1}$ at $x_{1}$, and the paths $\delta_{j}(j=2, \ldots, n)$ - formed by using the time reversed path $\gamma_{j}^{-1}$ the first half of the time, and $\gamma_{1}$ the second half - are the components of a path $\delta=\left(\delta_{1}, \ldots, \delta_{n}\right)$ in $p^{-1}\left(x_{1}\right)$ from $\delta(0)=\left(x_{1}, x_{2}, \ldots, x_{n}\right)$ to $\delta(1)=\left(x_{1}, x_{1}, \ldots, x_{1}\right)$. The continuity of $s_{i}$ implies that $\delta$ depends continuously on $\left(x_{1}, x_{2}, \ldots, x_{n}\right)$, so we have constructed a contraction of $A_{i}$ to $\left(x_{1}, x_{1}, \ldots, x_{1}\right)$ in $p^{-1}\left(x_{1}\right)$. Thus, $\operatorname{cat}\left(X^{n-1}\right) \leq \mathrm{TC}_{n}(X)$.

Remark 3.2 Using the fact that $\operatorname{cat}\left(X^{n}\right) \geq n$ if $X$ is not contractible (see Cornea, Lupton, Oprea and Tanré [3, Theorem 1.47]), we see that Proposition 3.1 recovers [23, Proposition 3.5].

Proposition 3.1 and (4) yield the following.

Corollary 3.3 For any path-connected space $X$,

$$
\operatorname{cat}\left(X^{n-1}\right) \leq \mathrm{TC}_{n}(X) \leq \operatorname{cat}\left(X^{n}\right) .
$$

We next show that the lower bound in Corollary 3.3 is optimal for topological groups. 
Proposition 3.4 For any path-connected topological group $G$,

$$
\mathrm{TC}_{n}(G) \leq \operatorname{cat}\left(G^{n-1}\right) .
$$

Proof Let $\epsilon$ denote the neutral element of $G$. Let $k=\operatorname{cat}\left(G^{n-1}\right)$ and choose an open covering $A_{0} \cup \cdots \cup A_{k}=G^{n-1}$, where each $A_{i}(i \in\{0, \ldots, k\})$ contracts in $G^{n-1}$ to an $(n-1)$-tuple $p_{i}$. Since $G$ is path-connected, each contracting homotopy can be extended to arrange that $p_{i}=(\epsilon, \ldots, \epsilon)=\epsilon^{(n-1)}$ for all $i=0, \ldots, k$.

Then, for $i \in\{0, \ldots, k\}$ set

$$
B_{i}=\left\{\left(g, g a_{2}, \ldots, g a_{n}\right) \mid\left(a_{2}, \ldots, a_{n}\right) \in A_{i}, g \in G\right\},
$$

which is open in $G^{n}$. We assert that $e_{n}^{G}$ admits a (continuous) section over each $B_{i}$. Indeed, for each $i$ the contractibility of $A_{i}$ in $G^{n-1}$ yields a path $\gamma_{a}$ in $G^{n-1}$ joining $\epsilon^{(n-1)}$ to each $a=\left(a_{2}, \ldots, a_{n}\right) \in A_{i} \subset G^{n-1}$ and depending continuously on $a \in A_{i}$. Augment $\gamma_{a}$ to a path $\gamma_{a}^{\prime}$ from $\epsilon^{(n)}$ to $\left(\epsilon, a_{2}, \ldots, a_{n}\right) \in B_{i}$ with the first coordinate remaining constant. Then, for any $g \in G, g \gamma_{a}^{\prime}$ is a path joining $(g, \ldots, g)=g \epsilon^{(n)} \in G^{n}$ to $\left(g, g a_{2}, \ldots, g a_{n}\right) \in B_{i}$ and depending continuously on $n$-tuples in $B_{i}$. Then, we get the required section

$$
s_{i}: B_{i} \rightarrow G^{J_{n}},
$$

where, on the $j^{\text {th }}$ interval of $J_{n}, s_{i}\left(g, g a_{2}, \ldots, g a_{n}\right)$ is the $j^{\text {th }}$ coordinate of $g \gamma_{a}^{\prime}$.

The proof will be complete once we check $B_{0} \cup \cdots \cup B_{k}=G^{n}$. Take $\left(b_{1}, \ldots, b_{n}\right) \in G^{n}$ and put $g=b_{1}$ and $a_{i}=g^{-1} b_{i}$. Then there exists $j$ such that $\left(a_{2}, \ldots, a_{n}\right) \in A_{j}$. So, $\left(b_{1}, \ldots, b_{n}\right) \in B_{j}$.

Corollary 3.3 and Proposition 3.4 combined yield the following.

Theorem 3.5 For any path-connected topological group $G$,

$$
\mathrm{TC}_{n}(G)=\operatorname{cat}\left(G^{n-1}\right) .
$$

Alternatively, we can look at the growth of $\mathrm{TC}_{n}$ in terms of the difference of any two consecutive values of $n$.

Corollary 3.6 Let $G$ be a path-connected topological group all of whose finite cartesian powers $G^{k}$ are normal. ${ }^{1}$ Then for $n \geq 3$,

$$
\mathrm{TC}_{n}(G)-\mathrm{TC}_{n-1}(G) \leq \operatorname{cat}(G) .
$$

${ }^{1}$ As noted in Section 2, we assume that a normal space is, by definition, Hausdorff. Thus, in view of the classical Birkhoff-Kakutani Theorem, the normality hypothesis in Corollary 3.6 holds when $G$ satisfies the first axiom of countability, ie provided $G$ is metrizable. 
Proof This is a consequence of Theorem 3.5 and the product inequality for the category, valid under the current normality assumptions in view of Proposition 2.2.

Unlike with topological groups, higher topological complexities of an arbitrary pathconnected space $X$ do not appear to be completely determined by the category of cartesian powers of $X$. Nonetheless, we can directly obtain the following bound on the difference of two consecutive higher topological complexities of $X$.

Proposition 3.7 Let $X$ be a path-connected space all of whose finite cartesian powers $X^{k}$ are normal. Then for $n \geq 3$,

$$
\mathrm{TC}_{n}(X)-\mathrm{TC}_{n-1}(X) \leq \operatorname{cat}\left(X^{2}\right) .
$$

Proof Use the argument in the proof of Corollary 3.6, replacing Theorem 3.5 by the inequalities in Corollary 3.3.

In particular $\mathrm{TC}_{n}(X)$ is bounded from above by a linear function on $n$ with slope cat $\left(X^{2}\right)$. According to [23, (5.1)], this slope can be improved to $\mathrm{TC}_{2}(X)$.

Next we consider the higher analogue of the usual cup length lower bound for TC. Recall that $d_{n}=d_{n}^{X}: X \rightarrow X^{n}$ stands for the iterated diagonal map. In the following definition we allow cohomology with local coefficients.

Definition 3.8 Given a space $X$ and a positive integer $n, \operatorname{cl}(X, n)$ denotes the cup length of elements in the kernel of the map induced in cohomology by $d_{n}^{X}$. Thus, $\operatorname{cl}(X, n)$ is the largest integer $m$ for which there exist cohomology classes $u_{i} \in H^{*}\left(X^{n} ; A_{i}\right)$ such that $d_{n}^{*} u_{i}=0$ for $i=1, \ldots, m$ and

$$
u_{1} \smile \cdots \smile u_{m} \neq 0 \in H^{*}\left(X^{n} ; A_{1} \otimes \cdots \otimes A_{m}\right) .
$$

The following result, which follows directly from [24, Theorems 4 and 5'], bounds $\mathrm{TC}_{n}(X)$ from below by $\operatorname{cl}(X, n)$, and from above by a ratio between the connectivity $\operatorname{conn}(X)$ and homotopy dimension $\operatorname{hdim}(X)$ of $X$, the latter being the smallest dimension of CW complexes having the homotopy type of $X$.

Theorem 3.9 For any path-connected space $X$ we have the inequalities

$$
\operatorname{cl}(X, n) \leq \operatorname{TC}_{n}(X) \leq \frac{n \operatorname{hdim}(X)}{\operatorname{conn}(X)+1} .
$$

We will also need the following bound on $\operatorname{cl}\left(X \times S^{k}, n\right)$ in terms of $\operatorname{cl}(X, n)$. 
Theorem 3.10 For any path-connected space $X$ and positive integers $n$ and $k$ we have $\operatorname{cl}\left(X \times S^{k}, n\right) \geq \operatorname{cl}(X, n)+n-1$. Provided $k$ is even and $H^{*}(X)$ is torsion free, this inequality can be improved to $\operatorname{cl}\left(X \times S^{k}, n\right) \geq \operatorname{cl}(X, n)+n$.

Proof Let $v$ be a generator of $H^{k}\left(S^{k}\right)=\mathbb{Z}$. Let $p_{i}:\left(S^{k}\right)^{n} \rightarrow S^{k}$ be the projection onto the $i^{\text {th }}$ factor and put $v_{i}=p_{i}^{*}(v)$ for $i=1, \ldots, n$. Assume that $\operatorname{cl}(X, n)=m$ and take $u_{1}, \ldots, u_{m}$ such that $d_{n}^{*}\left(u_{j}\right)=0$ for $j=1, \ldots, m$ and $u_{1} \smile \ldots \smile u_{m} \neq 0$. To prove the first assertion note that $d_{n}^{*}\left(v_{i}-v_{1}\right)=0$ for $i>1$, while the basis element $v_{2} \smile \cdots \smile v_{n} \in H^{*}\left(\left(S^{k}\right)^{n}\right)$ appears in the reduced expansion (using distributivity) of $\left(v_{2}-v_{1}\right) \smile \cdots \smile\left(v_{n}-v_{1}\right)$. Hence,

$$
u_{1} \smile \cdots \smile u_{m} \smile\left(v_{2}-v_{1}\right) \smile \cdots \smile\left(v_{n}-v_{1}\right) \neq 0 .
$$

Thus $\operatorname{cl}\left(X \times S^{k}, n\right) \geq \operatorname{cl}(X, n)+n-1$.

Assume $k$ is even and $H^{*}(X)$ is torsion free. Then $v_{1}+v_{2}+\cdots+v_{n-1}-(n-1) v_{n}$ lies in the kernel of $d_{n}^{*}$ and also has cup $n^{\text {th }}$ power which is equal to a nonzero multiple of $v_{1} \smile v_{2} \smile \cdots \smile v_{n}$. Hence,

$$
u_{1} \smile \cdots \smile u_{m} \smile\left(v_{1}+v_{2}+\cdots+v_{n-1}-(n-1) v_{n}\right)^{n} \neq 0 .
$$

Thus $\operatorname{cl}\left(X \times S^{k}, n\right) \geq \operatorname{cl}(X, n)+n$.

In [5] Farber obtained the subadditivity of $\mathrm{TC}_{2}$ under suitable topological hypothesis. The corresponding property for higher topological complexity is given next.

Proposition 3.11 Let $X$ and $Y$ be path-connected spaces. If $(X \times Y)^{n}$ is normal, then $\mathrm{TC}_{n}(X \times Y) \leq \mathrm{TC}_{n}(X)+\mathrm{TC}_{n}(Y)$.

Proof The natural homeomorphisms

$$
\begin{aligned}
(X \times Y)^{n} & \rightarrow X^{n} \times Y^{n}, \\
\left(\left(x_{1}, y_{1}\right), \ldots,\left(x_{n}, y_{n}\right)\right) & \mapsto\left(x_{1}, \ldots, x_{n}, y_{1}, \ldots, y_{n}\right), x_{i} \in X, y_{j} \in Y, \\
(X \times Y)^{J_{n}} & \rightarrow X^{J_{n}} \times Y^{J_{n}}, \\
\left(\varphi: J_{n} \rightarrow X \times Y\right) & \mapsto\left(\left(p_{X} \circ \varphi: J_{n} \rightarrow X\right),\left(p_{Y} \circ \varphi: J_{n} \rightarrow Y\right)\right),
\end{aligned}
$$

fit into the commutative diagram

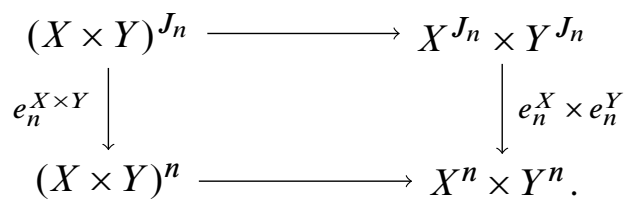

So, the desired conclusion follows directly from Proposition 2.2. 
As revealed in the case of spheres (next), Proposition 3.11 is optimal in general.

Corollary 3.12 $\mathrm{TC}_{n}\left(S^{k_{1}} \times S^{k_{2}} \times \cdots \times S^{k_{m}}\right)=m(n-1)+l$, where $l$ is the number of even dimensional spheres.

Proof Note that $\mathrm{TC}_{n}\left(S^{k}\right)=\operatorname{cl}\left(S^{k}, n\right)$ for all $k$ [23, Section 4]. Then the inequality $\operatorname{cl}\left(S^{k_{1}} \times \cdots \times S^{k_{m}}, n\right) \geq m(n-1)+l$ follows from Theorem 3.10 by induction, so $\mathrm{TC}_{n}\left(S^{k_{1}} \times \cdots \times S^{k_{m}}\right) \geq m(n-1)+l$ by Theorem 3.9. The opposite estimate follows from Proposition 3.11.

The calculation of the higher topological complexity of the $k$-dimensional torus $T^{k}=\left(S^{1}\right)^{k}$, partially solved for $k=2$ in [23, Proposition 5.1], is a consequence of either Corollary 3.12 or Theorem 3.5.

Corollary 3.13 We have $\mathrm{TC}_{n}\left(T^{k}\right)=k(n-1)$.

Theorem 3.14 Let $X$ be a $C W$ complex of finite type, and $R$ a principal ideal domain. Take $u \in H^{d}(X ; R)$ with $d>0, d$ even, and assume that the $n$-fold iterated self $R$-tensor product $u^{m} \otimes \cdots \otimes u^{m} \in\left(H^{m d}(X ; R)\right)^{\otimes n}$ is an element of infinite additive order. Then $\mathrm{TC}_{n}(X) \geq m n$.

Proof For $i=1, \ldots, n$, let $p_{i}: X^{n} \rightarrow X$ be the projection onto the $i^{\text {th }}$ factor and put $u_{i}=p_{i}^{*}(u) \in H^{d}\left(X^{n} ; R\right)$. In view of Theorem 3.9, the our inequality follows from

$$
v:=\left(u_{2}-u_{1}\right)^{2 m}\left(u_{3}-u_{1}\right)^{m} \cdots\left(u_{n}-u_{1}\right)^{m} \neq 0 .
$$

In order to check (5), note that $v$ comes from the tensor product, which injects into the cohomology of the cartesian product by the Künneth Theorem (this is where the finiteness hypotheses are used). So, calculations can be performed in the former $R$-module. Now, assuming that $\operatorname{dim}(X) \leq d m+1$, we have

$$
\begin{aligned}
& v=\left(u_{2}-u_{1}\right)^{2 m}\left(u_{3}-u_{1}\right)^{m} \cdots\left(u_{n}-u_{1}\right)^{m} \\
&=(-1)^{m}\left(\begin{array}{c}
2 m \\
m
\end{array}\right) u_{1}^{m} u_{2}^{m}\left(u_{3}-u_{1}\right)^{m} \cdots\left(u_{n}-u_{1}\right)^{m} \\
&=(-1)^{m}\left(\begin{array}{c}
2 m \\
m
\end{array}\right) u_{1}^{m} u_{2}^{m} u_{3}^{m}\left(u_{4}-u_{1}\right)^{m} \cdots\left(u_{n}-u_{1}\right)^{m} \\
& \vdots \\
&=(-1)^{m}\left(\begin{array}{c}
2 m \\
m
\end{array}\right) u_{1}^{m} u_{2}^{m} \cdots u_{n}^{m},
\end{aligned}
$$

which is nonzero by hypothesis. For $\operatorname{dim}(X)$ arbitrary, consider the skeletal inclusion $j: X^{(d m+1)} \rightarrow X$ and note that $v \neq 0$ since $j^{*}(v) \neq 0$. 
Corollary 3.15 For every closed simply connected symplectic manifold $M^{2 m}$ we have $\mathrm{TC}_{n}(M)=n m$.

Proof This follows from Theorem 3.14 (taking $u$ to be the cohomology class given by the symplectic 2-form on $M$, and noting that the hypothesis on $u^{m} \otimes \cdots \otimes u^{m}$ holds since the coefficients are taken over the reals), inequality (4), the product inequality for category and the inequality $\operatorname{cat}\left(M^{2 m}\right) \leq m$ which follows from [24, Theorem 5 , page 75]. (This argument also yields $\operatorname{cat}\left(M^{2 m}\right)=m$, a well known fact.)

Of course, Corollary 3.15 applies to complex projective spaces. In the quaternionic case essentially the same proof gives the following.

Corollary 3.16 The quaternionic projective space of real dimension $4 m, \mathbb{H} \mathrm{P}^{m}$, has $\mathrm{TC}_{n}\left(\mathbb{H} \mathrm{P}^{m}\right)=n m$.

Note that Corollaries 3.15 and 3.16 imply that the upper bound in Corollary 3.3 as well as both bounds in Theorem 3.9 are optimal in general.

\section{Symmetric topological complexity}

In this section we introduce two symmetric versions of $\mathrm{TC}_{n}$. One of them, $\mathrm{TC}_{n}^{\Sigma}$, has the advantage of being a homotopy invariant. The other, $\mathrm{TC}_{n}^{S}$, gives (up to our normalization convention) the natural generalization of the symmetric topological complexity studied by Farber and Grant in [9]. We begin with the $n=2$ case of the homotopically well-behaved version.

Consider the involutions $\tau: X \times X \rightarrow X \times X$ and $\bar{\tau}: P(X) \rightarrow P(X)$ defined by $\tau(x, y)=(y, x)$ and $\bar{\tau}(\gamma)(t)=\gamma(1-t)$, for $(x, y) \in X \times X$ and $\gamma \in P(X)$. We work with symmetric subsets $A \subseteq X \times X$ (ie those for which $\tau A=A$ ) and equivariant maps $s: A \rightarrow P(X)$ (ie those satisfying $\bar{\tau}(s(a))=s(\tau(a)$ ) for all $a \in A)$.

Definition 4.1 We have $\mathrm{TC}^{\Sigma}(X)$ is the least integer $k$ which satisfies $X \times X=$ $A_{0} \cup A_{1} \cup \cdots \cup A_{k}$, where each $A_{i}$ is open, symmetric and admits a continuous equivariant section $s_{i}: A_{i} \rightarrow P(X)$ of the map $e_{2}$ in (2).

Before proving (in Proposition 4.7 below) that $\mathrm{TC}^{\Sigma}(X)$ is a homotopy invariant of $X$, we show that its numerical value differs by at most one from the numerical value of Farber and Grant's symmetric topological complexity. In accordance with the normalization hypothesis in this paper, we must compare $\operatorname{TC}^{\Sigma}(X)$ with

$$
\mathrm{TC}_{2}^{S}(X)=\mathfrak{g e n u s}\left(\varepsilon_{2}\right)+1,
$$


where $\varepsilon_{2}$ is the map on the right-hand side in (3). Note that, under the perspective of [9], the " +1 " summand in (6) is meant to take into account the obvious equivariant section of $e_{2}$ on the diagonal.

Proposition 4.2 For each Euclidean neighborhood retract (ENR) $X$ we have

$$
\operatorname{TC}_{2}^{S}(X)-1 \leq \mathrm{TC}^{\Sigma}(X) \leq \mathrm{TC}_{2}^{S}(X) .
$$

Remark 4.3 We will prove a more general version of Proposition 4.2 (Theorem 4.8 below). The proof of the general version is considerably more elaborate as it requires a rather involved use of equivariant Euclidean neighborhood retracts. For the sake of clarity, we offer first a much simpler argument proving Proposition 4.2.

Proof of Proposition 4.2 To prove the first inequality, take an open covering $X \times X=$ $A_{0} \cup \cdots \cup A_{k}$, where each $A_{i}$ is symmetric and has a continuous equivariant section of $e_{2}$. The $\mathbb{Z} / 2$-action $\tau$ on $X \times X$ yields the orbit map $\rho_{2}: X \times X \rightarrow(X \times X) / \tau$. Then, for each $i=0, \ldots, k, \rho_{2}\left(A_{i}-d_{2}(X)\right)$ is open and has a section of $\varepsilon_{2}$, and thus $\mathfrak{g e n u s}\left(\varepsilon_{2}\right) \leq \mathrm{TC}^{\Sigma}(X)$.

For the second inequality, take $B_{0}, \ldots, B_{l}$, with $B_{0} \cup \cdots \cup B_{l}=\rho_{2}\left(X \times X-d_{2}(X)\right)$, where each $B_{i}$ is open and has a section of $\varepsilon_{2}$. Then each $\rho_{2}^{-1}\left(B_{i}\right)$ is symmetric, open in $X \times X$ and admits an equivariant section of $e_{2}$, cf [9, Lemma 8]. Further, since $X$ is an ENR, there is a symmetric open neighborhood of $d_{2}(X)$ supporting an equivariant section of $e_{2}$; see the proof of [9, Corollary 9]. Consequently we have that $\operatorname{TC}^{\Sigma}(X) \leq 1+\mathfrak{g e n u s}\left(\varepsilon_{2}\right)$.

The two examples below show that both bounds in Proposition 4.2 are optimal in general.

Example 4.4 For $X$ contractible, $\operatorname{TC}_{2}(X)=\mathrm{TC}^{\Sigma}(X)=0$ while $\mathrm{TC}_{2}^{S}(X)=1$. Indeed, take a point $x_{0} \in X$ and a contraction $H: X \times I \rightarrow X$, with $H(x, 0)=x$ and $H(x, 1)=x_{0}$ for all $x \in X$. Given $(a, b) \in X \times X$, take the path $\sigma=s(a, b): I \rightarrow X$ such that $\sigma(t)=H(a, 2 t)$ for $0 \leq t \leq \frac{1}{2}$ and $\sigma(t)=H(b, 2-2 t)$ for $\frac{1}{2} \leq t \leq 1$. Then $s$ is an equivariant section for $e_{2}^{X}$ and, in view of the general inequality

$$
\mathrm{TC}_{2}(X) \leq \mathrm{TC}^{\Sigma}(X),
$$

this gives $\mathrm{TC}_{2}(X)=\mathrm{TC}^{\Sigma}(X)=0$. The same argument, but now using (6), gives $\operatorname{TC}_{2}^{S}(X)=1$; see [10, Example 7]. 
Example 4.5 The numbers $\mathrm{TC}_{2}^{S}\left(S^{k}\right)$ and $\mathrm{TC}_{2}\left(S^{k}\right)$ have been computed in [9, Corollary 18] and [5], respectively. Here we use the inequalities $\mathrm{TC}_{2} \leq \mathrm{TC}^{\Sigma} \leq \mathrm{TC}^{S}$ together with the fact that $\mathrm{TC}_{2}^{S}\left(S^{k}\right)=2=\mathrm{TC}_{2}\left(S^{2 k}\right)$ to deduce $\mathrm{TC}^{\Sigma}\left(S^{2 k}\right)=\mathrm{TC}_{2}^{S}\left(S^{2 k}\right)=2$ for all $k$. On the other hand, since $\mathrm{TC}_{2}\left(S^{2 k+1}\right)=1$, the above argument only gives $1 \leq \mathrm{TC}^{\Sigma}\left(S^{2 k+1}\right) \leq \mathrm{TC}_{2}^{S}\left(S^{2 k+1}\right)=2$. Incidentally, note that the construction in [8, Example 4.8] gives an open covering $S^{2 k+1} \times S^{2 k+1}=A_{0} \cup A_{1}$ by symmetric sets $A_{i}$, and continuous sections of $e_{2}$ over each $A_{i}, i=0,1$. However, one of these sections is not equivariant, which prevents us from deducing $\operatorname{TC}^{\Sigma}\left(S^{k}\right)=1$.

We next define higher analogues of $\mathrm{TC}^{\Sigma}$. Recall that for a given $n$, the symmetric group $\Sigma_{n}$ acts on the right of $X^{n}$ and $X^{J_{n}}$ by permuting coordinates and paths, respectively. Further, the fibration $e_{n}$ in (2) is $\Sigma_{n}$-equivariant. We now work with symmetric subsets $A \subseteq X^{n}$ (ie those for which $A \sigma=A$ for all $\sigma \in \Sigma_{n}$ ) and equivariant maps $s: A \rightarrow X^{J_{n}}$ (ie those satisfying $s(a) \sigma=s(a \sigma)$ for all $a \in A$ and $\sigma \in \Sigma_{n}$ ). Definition 4.1 can now be extended to the following.

Definition 4.6 We have that $\operatorname{TC}_{n}^{\Sigma}(X)$ is the least integer $k$ which satisfies $X^{n}=$ $A_{0} \cup A_{1} \cup \cdots \cup A_{k}$, where each $A_{i}$ is open, symmetric and admits a continuous equivariant section $s_{i}: A_{i} \rightarrow X^{J_{n}}$ for the map $e_{n}$ in (2).

Proposition 4.7 We have $\operatorname{TC}_{n}^{\Sigma}(X)$ is a homotopy invariant of $X$.

Proof It suffices to prove that, given $f: Y \rightarrow X$ and $g: X \rightarrow Y$ with $g f \simeq 1_{Y}$, we have $\operatorname{TC}_{n}^{\Sigma}(X) \geq \operatorname{TC}_{n}^{\Sigma}(Y)$ for all $n$. Let $H: 1_{Y} \simeq g f$ be a homotopy $H: Y \times[0,1] \rightarrow Y$ such that $H(y, 0)=y$ and $H(y, 1)=g f(y)$.

Let $A$ be an open symmetric subset of $X^{n}$, and let $s: A \rightarrow X^{J_{n}}$ be an equivariant section of $e_{n}^{X}$ over $A$. Given $a=\left(a_{1}, \ldots, a_{n}\right) \in A$, let $s_{i}(a)$ denote the restriction of $s(a) \in X^{J_{n}}$ to the $i^{\text {th }}$ wedge summand of $J_{n}$ (this is a path in $X$ joining $x_{0}$ and $a_{i}$ for some $x_{0} \in X$ that depends continuously on $a$ ). Note that the equivariance of $s$ gives

$$
s_{i}\left(a_{\sigma(1)}, \ldots, a_{\sigma(n)}\right)=s_{\sigma(i)}\left(a_{1}, \ldots, a_{n}\right) \text { for } \sigma \in \Sigma_{n} .
$$

Take $B:=\left(f^{n}\right)^{-1}(A)$, where $f^{n}$ stands for the $n^{\text {th }}$ cartesian power of $f$, and consider the map $s^{\prime}: B \rightarrow Y^{J_{n}}$ which, at a given $b \in B$ such that $f^{n}(b)=a$, has $s_{i}^{\prime}(b):=\left(g \circ s_{i}(a)\right) \cdot \gamma_{i}$ as its restriction to the $i^{\text {th }}$ wedge summand of $J_{n}$, where $\gamma_{i}$ is the path in $Y$ given by

$$
\gamma_{i}(t)=H\left(b_{i}, 1-t\right) .
$$

Then, $s^{\prime}$ is an equivariant continuous section of $e_{n}^{Y}$ over $B$ in view of (7). 
In this setting, if $X=A_{0} \cup \cdots \cup A_{k}$, where each $A_{j}(j=0, \ldots, k)$ is open, symmetric and admits a continuous equivariant section of $e_{n}^{X}$, then $Y=B_{0} \cup \cdots \cup B_{k}$ where each $B_{j}$ - defined as above using $A_{j}$ - is open, symmetric, and admits a continuous equivariant section of $e_{n}^{Y}$. Hence, $\mathrm{TC}_{n}^{\Sigma}(X) \geq \mathrm{TC}_{n}^{\Sigma}(Y)$.

The following assertion is our higher analogue of Proposition 4.2.

Theorem 4.8 If $X$ is an ENR, and $\varepsilon_{n}$ is the map on the right-hand side in (3), then

$$
\mathfrak{g e n u s}\left(\varepsilon_{n}\right) \leq \mathrm{TC}_{n}^{\Sigma}(X) \leq \mathfrak{g e n u s}\left(\varepsilon_{n}\right)+\cdots+\mathfrak{g e n u s}\left(\varepsilon_{2}\right)+n-1 .
$$

The first inequality in (8) follows just as in the proof of Proposition 4.2: if $e_{n}$ admits an equivariant section over $A \subset X^{n}$, then $\varepsilon_{n}$ admits a section over $\rho_{n}\left(A \cap \operatorname{Conf}_{n}(X)\right)$, where $\rho_{n}: X^{n} \rightarrow X^{n} / \Sigma_{n}$ stands for the canonical projection. Our efforts will therefore focus on the second inequality in (8), whose proof requires some preparation.

Definition 4.9 A topological space $X$ with an action of a compact Lie group $G$ is called a Euclidean neighborhood $G$-retract $(G$-ENR) if $X$ can be $G$-equivariantly embedded, as a $G$-equivariant retract of a $G$-symmetric neighborhood of $X$, into an orthogonal representation of $G$.

In what follows we will make implicit use of the following fact: if a $G$-ENR $X$ is $G$-equivariantly embedded in a given orthogonal representation $\mathbb{R}^{N}$ of $G$, then there exists a $G$-symmetric neighborhood $U$ of $X$ in $\mathbb{R}^{N}$ and a $G$-equivariant retraction $U \rightarrow X$. As noticed at the end of the introduction in Jaworowski [17], such a property follows by applying the equivariant version of the Tietze Theorem (Tietze-Gleason Theorem; see Bredon [2] and Gleason [14]) to the nonequivariant argument by Dold in [4, Proposition and Definition IV.8.5].

We shall use the following weaker version of [17, Theorem 2.1]. ${ }^{2}$

Theorem 4.10 (Jaworowski) Let $L$ be a finite group acting on an ENR $Z$. Then $Z$ is an $L-E N R$ if for every subgroup $G$ of $L$, the fixed point set $Z^{G}$ is an ENR.

Next, consider the $\Sigma_{n}$-equivariant filtration

$$
d_{n}(X)=D^{1}(X) \subset \cdots \subset D^{n-1}(X) \subset D^{n}(X)=X^{n},
$$

${ }^{2}$ Although Jaworowski's theorem was originally set in terms of a combination of the concepts of ANR's and ENR's, for our formulation the reader should keep in mind the fact that any ENR is an ANR (which is elementary in view of the Tietze Theorem). 
where, for $i \in\{1, \ldots, n\}$, we have that $D^{i}(X)$ is the closed set consisting of the $n-$ tuples $\left(x_{1}, x_{2}, \ldots, x_{n}\right)$ such that the $\operatorname{set}\left\{x_{1}, x_{2}, \ldots, x_{n}\right\}$ has cardinality at most $i$. For instance, $D^{n-1}(X)$ is the so-called fat diagonal in $X^{n}$, otherwise denoted by $\Delta_{n}(X)$. Compare the filtration in (9) with the one considered in Kallel [18, Section 1].

Set $D^{0}(X)=\varnothing$, and for $1 \leq i \leq n$ let $C^{i}$ stand for the difference $D^{i}(X)-D^{i-1}(X)$, the subspace of $n$-tuples $\left(x_{1}, x_{2}, \ldots, x_{n}\right)$ such that the set $\left\{x_{1}, x_{2}, \ldots, x_{n}\right\}$ has cardinality $i$. Note that $C^{n}=\operatorname{Conf}_{n}(X)$ and that for $i<n$, each partition $\mathcal{P}=\left\{P_{1}, \ldots, P_{i}\right\}$ of $\{1,2, \ldots, n\}$ into $i$ nonempty sets determines a closed subspace $C_{\mathcal{P}}^{i} \subset C^{i}$ formed by those tuples $\left(x_{1}, \ldots, x_{n}\right)$ in $C^{i}$ satisfying $x_{r}=x_{s}$ whenever both $r$ and $s$ lie in the same part $P_{j}$ for some $j$.

Note that $C^{i}$ is the disjoint union of the $C_{\mathcal{P}}^{i}$ 's, each of which maps homeomorphically onto $\operatorname{Conf}_{i}(X)$ under a suitable coordinate projection. (For instance, for $n=3$ the three closed subspaces partitioning $C^{2}$ are determined by the three requirements $x_{1}=x_{2}, x_{1}=x_{3}$, and $x_{2}=x_{3}$; in the latter case, the required projection can be chosen to be $\left(x_{1}, x_{2}, x_{3}\right) \mapsto\left(x_{1}, x_{2}\right)$.) Therefore, we have a continuous (surjective) map $\pi_{i}: C^{i} \rightarrow \operatorname{Conf}_{i}(X)$.

Let $P^{i}$ denote the subspace of $e_{n}^{-1}\left(C^{i}\right)$ consisting of those multipaths $\alpha=\left\{\alpha_{i}\right\}_{i=1}^{n}$ satisfying $\alpha_{k}=\alpha_{\ell}$ whenever $\alpha_{k}\left(1_{k}\right)=\alpha_{\ell}\left(1_{\ell}\right)$. Proceeding as above, we get a continuous surjection $\Pi_{i}: P^{i} \rightarrow e_{i}^{-1}\left(\operatorname{Conf}_{i}(X)\right)$ in such a way that in the commutative diagram

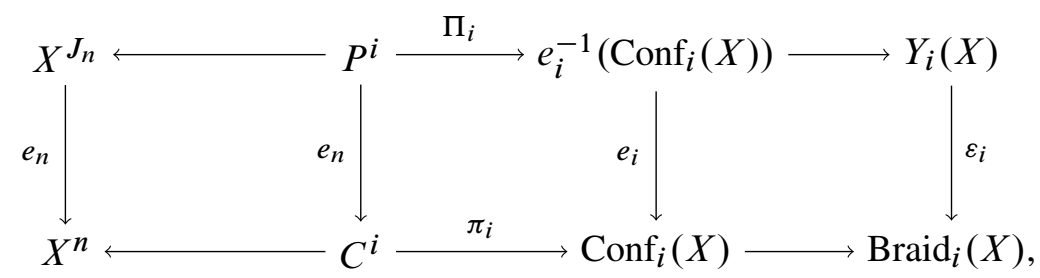

the second and third squares are pullbacks, and the two leftmost horizontal maps are inclusions but do not determine a pullback square.

Our last ingredient in preparation for the proof of (8) is given by taking an arbitrary open subset $W$ of $\operatorname{Braid}_{i}(X)$. We then let $A=\pi_{i}^{-1}\left(W^{\prime}\right)$, where $W^{\prime}$ stands for the inverse image of $W$ under the projection $\operatorname{Conf}_{i}(X) \rightarrow \operatorname{Braid}_{i}(X)$. Clearly $W^{\prime}$ is $\Sigma_{i}$-symmetric and $A$ is $\Sigma_{n}$-symmetric. This setup will be in force in the following two auxiliary results, which are the basis of our proof of the second inequality in (8).

Lemma 4.11 The space $A$ is a $\Sigma_{n}-E N R$. 
Proof Note first that every $C_{\mathcal{P}}^{i}$ is an ENR, because it is homeomorphic to $\operatorname{Conf}_{i}(X)$ which, in turn, is an open subset of the ENR $X^{i}$. Now, every $g \in \Sigma_{n}$ yields a homeomorphism from any given $C_{\mathcal{P}}^{i}$ onto some $C_{\mathcal{P}^{\prime}}^{i}$. In particular for $\mathcal{P}=\mathcal{P}^{\prime}$, if there is some $x \in C_{\mathcal{P}}^{i}$ fixed by $g$, then $y \cdot g=y$ for all $y \in C_{\mathcal{P}}^{i}$, ie $\left(C_{\mathcal{P}}^{i}\right)^{g}=C_{\mathcal{P}}^{i}$. Hence, for any subgroup $G$ of $\Sigma_{n}$, the set $\left(C_{\mathcal{P}}^{i}\right)^{G}$ is either empty or the whole of $C_{\mathcal{P}}^{i}$, and therefore an ENR. Consequently, $\left(C^{i}\right)^{G}$ is an ENR since $C^{i}$ is the disjoint union of the various $C_{\mathcal{P}}^{i}$ 's, and $A^{G}$ is an ENR since $A$ is open in $C^{i}$. Thus, by Theorem 4.10, $A$ is a $\Sigma_{n}$-ENR, as asserted.

Lemma 4.12 Assume $s: A \rightarrow P^{i}$ is a $\Sigma_{n}$-equivariant section of the second vertical map in (10). Then there is a $\Sigma_{n}$-symmetric neighborhood $U$ of $A$ in $X^{n}$ that admits a $\Sigma_{n}$-equivariant section $\sigma: U \rightarrow X^{J_{n}}$ of the first vertical map in (10).

Proof We begin by noticing that, as a consequence of Theorem 4.10, $X^{n}$ is a $\Sigma_{n}$-ENR. Indeed, for any subgroup $G$ of $\Sigma_{n}$, the fixed point set of $G$ on $X^{n}$ is an intersection of hyperplanes $x_{i}=x_{j}$ in $X^{n}$. Hence, $\left(X^{n}\right)^{G}$ is an ENR since it is homeomorphic to $X^{m}$ for $m \leq n$. Thus, we can take $\Sigma_{n}$-equivariant embeddings $A \rightarrow X^{n} \rightarrow \mathbb{R}^{N}$, and a $\Sigma_{n}$-equivariant retraction $r^{\prime}: O \rightarrow A$ of a $\Sigma_{n}$-symmetric neighborhood $O$ of $A$ in $\mathbb{R}^{N}$, where $\mathbb{R}^{N}$ is an orthogonal representation of $\Sigma_{n}$.

Set $V=O \cap X^{n}$. Then $V$ is a $\Sigma_{n}$-symmetric neighborhood of $A$ in $X^{n}$, and $r=\left.r^{\prime}\right|_{V}: V \rightarrow A$ is a $\Sigma_{n}$-equivariant retraction. Note that $V$ is an open $\Sigma_{n}$-symmetric subset of the $\Sigma_{n}-$ ENR $X^{n}$, and so $V$ is a $\Sigma_{n}$-ENR too. We can then choose an open $\Sigma_{n}$-symmetric neighborhood $Y$ of $V$ in $\mathbb{R}^{N}$, and a $\Sigma_{n}$-equivariant retraction $\rho: Y \rightarrow V$. Let $U \subset V$ consist of all points $v \in V$ such that the segment from $v$ to $i \circ r(v)$ lies in $Y$ where $i$ stands for the inclusion $A \hookrightarrow V$ (cf [4, Corollary IV.8.7]). Clearly $U$ is a neighborhood of $A$ in $V$, and hence in $X^{n}$. Furthermore, the composition $\left.i \circ r\right|_{U}$ and the inclusion $U \hookrightarrow V$ are homotopic via the homotopy

$$
\Phi: U \times I \rightarrow V, \quad \Phi(u, t)=\rho(t \cdot u+(1-t) \cdot i \circ r(u)) .
$$

Note that $U$ is $\Sigma_{n}$-symmetric and $\Phi$ is $\Sigma_{n}$-equivariant, since the $\Sigma_{n}$-action on $\mathbb{R}^{N}$ is orthogonal and so it maps lines to lines.

We use the homotopy $\Phi$ in order to construct a $\Sigma_{n}$-equivariant section $\sigma: U \rightarrow X^{J_{n}}$ of the first vertical map in (10). For $x \in U$, consider the path $\beta: I \rightarrow V, \beta(t)=\Phi(x, t)$, starting at $y=\beta(0)=r(x) \in A$ and ending at $x$. Since $V$ is a subset of $X^{n}$, we can set $x=\left(x_{1}, \ldots, x_{n}\right), y=\left(y_{1}, \ldots, y_{n}\right)$, and $\beta=\left(\beta_{1}, \ldots, \beta_{n}\right)$, so each $\beta_{i}$ is a path in $X$ from $y_{i}$ to $x_{i}$. Further, $s(y)$ gives a multipath $\left\{\alpha_{i}\right\}_{i=1}^{n}$ with $\alpha_{i}(1)=y_{i}$ and $\alpha_{i}(0)=\alpha_{j}(0)$ for all $1 \leq i, j \leq n$. Then the multipath $\left\{\alpha_{i} \cdot \beta_{i}\right\}_{i=1}^{n}$ determines an element $\sigma(x) \in X^{J_{n}}$ with $e_{n}(\sigma(x))=x$. This defines the required $\Sigma_{n}$-equivariant section over $U$. 
Note that the commutativity of the two pullback squares in (10) imply that the hypothesis in Lemma 4.12 holds whenever $W$ (the arbitrary open subset of $\operatorname{Braid}_{i}(X)$ taken in the paragraph previous to Lemma 4.11) is chosen to admit a section of the fourth vertical map in (10). Thus we obtain the following.

Proof of Theorem 4.8 (conclusion) In view of Lemmas 4.11 and 4.12 we can choose $1+\mathfrak{g e n u s}\left(\varepsilon_{i}\right) \Sigma_{n}$-equivariant local sections for $e_{n}$ whose domains cover $C^{i}$, and thus a total of

$$
\sum_{i=2}^{n}\left(1+\mathfrak{g e n u s}\left(\varepsilon_{i}\right)\right)+1=\mathfrak{g e n u s}\left(\varepsilon_{n}\right)+\cdots+\mathfrak{g e n u s}\left(\varepsilon_{2}\right)+n
$$

$\Sigma_{n}$-equivariant local sections for $e_{n}$ whose domains cover $X^{n}$. Here the " +1 " on the left-hand side in (11) accounts for the obvious equivariant section on the diagonal $D^{1}(X)$. The theorem follows.

A comparison of Proposition 4.2 and Theorem 4.8 suggests the following generalization of (6).

Definition 4.13 For $n \geq 2$ set

$$
\operatorname{TC}_{n}^{S}(X)=\mathfrak{g e n u s}\left(\varepsilon_{n}\right)+\cdots+\mathfrak{g e n u s}\left(\varepsilon_{2}\right)+n-1 .
$$

This is a minor variation of the one proposed in the short final section in [23], and will be explored next for $X$ a sphere.

\section{5 Švarc genus of $\varepsilon_{n}$ and configuration spaces of spheres}

The following result, which is a specialization of [24, Theorem 5, page 75] (recalling that $(\Omega X)^{n-1}$ is the homotopy fiber of the map $\varepsilon_{n}=\varepsilon_{n}^{X}: Y_{n}(X) \rightarrow \operatorname{Braid}_{n}(X)$ in (3)), gives a general upper bound for $\mathfrak{g e n u s}\left(\varepsilon_{n}\right)$ analogous to that in Theorem 3.9.

Proposition 5.1 If $X$ is an $(s-1)$-connected space and $\operatorname{Braid}_{n}(X)$ has the homotopy type of a $d$-dimensional CW space, then $\mathfrak{g e n u s}\left(\varepsilon_{n}\right) \leq d / s$.

For instance, $\mathfrak{g e n u s}\left(\varepsilon_{n}^{X}\right)=0$ for any contractible space $X$. This generalizes the phenomenon noted in Example 4.4. Part of the goal of this section is to show that the bound in Proposition 5.1 becomes an equality in some concrete situations, other than those noted for a contractible space $X$. Yet, the following considerations are written in conjectural terms; nonconjectural statements start from equation (14) on. 
The conjectural inequality in (1) is based on Proposition 5.1. To illustrate the idea, start by recalling from Example 4.5 the equality $\mathrm{TC}_{2}^{S}\left(S^{k}\right)=2$ valid for any $k$. Farber and Grant prove that $\mathrm{TC}_{2}^{S}\left(S^{k}\right)$ is no greater than 2 by producing a symmetric motion planner with two local rules. Their construction makes use of a well-known explicit $\Sigma_{2}$-equivariant deformation retraction $\operatorname{Conf}_{2}\left(S^{k}\right) \rightarrow S^{k}$ that implies a corresponding homotopy equivalence

$$
\operatorname{Braid}_{2}\left(S^{k}\right) \simeq \mathbb{R} \mathrm{P}^{k}
$$

Here we note that Proposition 5.1 gives an alternative direct way to deduce the inequality $\mathrm{TC}_{2}^{S}\left(S^{k}\right) \leq 2$ : all that is needed is the fact that $\operatorname{hdim}\left(\operatorname{Braid}_{2}\left(S^{k}\right)\right)=k$. In order to extend this simple argument for higher $\mathrm{TC}_{n}^{S}$ we would need to have a good hold on the homotopy dimension of $\operatorname{Braid}_{n}\left(S^{k}\right)$. Remark 5.3 below provides evidence toward the following.

Conjecture 5.2 For $n \geq 2$ and $k \geq 1$, $\operatorname{hdim}\left(\operatorname{Braid}_{n}\left(S^{k}\right)\right)=(k-1)(n-1)+1$.

Remark 5.3 Note that the validness of Conjecture 5.2 for $n=2$ follows from (12). Likewise, the case $k=1$ of Conjecture 5.2 is well known: $\operatorname{Braid}_{n}\left(S^{1}\right)$ has the homotopy type of $S^{1}$ (cf [18, Proposition 2.5]). On the other hand, from the calculations of homology groups in Feichtner and Ziegler [13], it can be proved that Conjecture 5.2 is true if $\operatorname{Braid}_{n}\left(S^{k}\right)$ is replaced by $\operatorname{Conf}_{n}\left(S^{k}\right)$ when $k \geq 3$. At any rate, since the homotopy dimension of a space is not less than the homotopy dimension of any of its covering spaces, we have

$$
\operatorname{hdim}\left(\operatorname{Braid}_{n}\left(S^{k}\right)\right) \geq \operatorname{hdim}\left(\operatorname{Conf}_{n}\left(S^{k}\right)\right)=(k-1)(n-1)+1 .
$$

Therefore the crux of the matter in settling Conjecture 5.2 (and, as a consequence, the equality $\left.\operatorname{hdim}\left(\operatorname{Conf}_{n}\left(S^{k}\right)\right)=(n-1)(k-1)+1\right)$ rests in producing a CW complex of dimension $(k-1)(n-1)+1$ which has the $\Sigma_{n}$-equivariant homotopy type of $\operatorname{Conf}_{n}\left(S^{k}\right)$. The second and fourth authors of this paper have an ongoing project aiming at such a goal; the basic ideas have been presented by the authors in the second half of [1]. However, it turns out that those ideas require an important tuning and have actually become a completely independent paper (which will appear elsewhere). The present paper then focuses on the first half of [1], ie the development of the properties of the sequential topological complexity.

We have mentioned that the validness of (1) would follow from Conjecture 5.2. In fact, in view of Proposition 5.1, we see that Conjecture 5.2 would actually imply the validness of the more detailed but still conjectural estimate

$$
\mathfrak{g e n u s}\left(\varepsilon_{i}\right) \leq i-1-\frac{i-2}{k} \text { for } X=S^{k} \text { and } i \geq 2 .
$$


The remainder of the section is devoted to presenting evidence for the validness and general optimality of (13).

We have observed that (13) holds true for $i=2$. As for its optimality, it is worth observing that Farber and Grant prove in [9, Section 3] the inequality

$$
\operatorname{TC}_{2}^{S}\left(S^{k}\right) \geq 2
$$

by means of an involved extension of Haefliger's calculation of the mod 2 cohomology ring $H^{*}\left(\operatorname{Braid}_{2}(M) ; \mathbb{Z} / 2\right)$ for $M$ a closed smooth manifold. But a simpler argument is available. Start by observing that if (14) were to fail, then there would exist a continuous section $\sigma$ for $\varepsilon_{2}^{S^{k}}$. In such a situation we could consider the composite

$$
S^{k} \stackrel{\alpha}{\rightarrow} \operatorname{Conf}_{2}\left(S^{k}\right) \stackrel{\widetilde{\sigma}}{\rightarrow} e_{2}^{-1}\left(\operatorname{Conf}_{2}\left(S^{k}\right)\right) \hookrightarrow P S^{k},
$$

where $\alpha(x)=(x,-x)$ and $\tilde{\sigma}$ would be the (Z/Z -equivariant) pullback of $\sigma$ under (3). The adjoint of this composite would then yield a homotopy $H: S^{k} \times[0,1] \rightarrow S^{k}$ between the identity $H(-, 0)$ and the antipodal map $H(-, 1)$, and which would in addition satisfy the relation

$$
H(x, t)=H(-x, 1-t) .
$$

But this is impossible since the identity on $S^{k}$ (which has degree 1) cannot be homotopic to the presumed map $H\left(-, \frac{1}{2}\right)$ which, in view of (15), would factor as

$$
S^{k} \stackrel{\text { proj }}{\longrightarrow} \mathbb{R P}^{k} \rightarrow S^{k},
$$

and would therefore have even degree.

The above argument, as well as the closely related proof of Proposition 5.4 below, were pointed out to the authors by Peter Landweber.

Proposition 5.4 Let $k$ be a positive odd integer. For $X=S^{k}$ and $i \geq 2, \mathfrak{g e n u s}\left(\varepsilon_{i}\right) \geq 1$. Further, $\mathfrak{g e n u s}\left(\varepsilon_{i}\right)=1$ provided $k=1$.

Proof of Proposition 5.4 The second assertion follows from the first one in view of Proposition 5.1 and the first part of Remark 5.3. To prove the first assertion, we derive a contradiction from the assumption that $\varepsilon_{i}$ admits a global continuous section $\sigma$. Consider the map $c: S^{k} \rightarrow\left(S^{k}\right)^{J_{i}}$ given as the composite

$$
S^{k} \stackrel{\alpha}{\rightarrow} \operatorname{Conf}_{i}\left(S^{k}\right) \stackrel{\widetilde{\sigma}}{\rightarrow} e_{i}^{-1}\left(\operatorname{Conf}_{i}\left(S^{k}\right)\right) \hookrightarrow\left(S^{k}\right)^{J_{i}} .
$$

Here $\alpha(x)=\left(x, z x, z^{2} x, \ldots, z^{i-1} x\right)$, where $z \in S^{1}$ is a primitive $i^{\text {th }}$ root of unity acting on $S^{k}$ in the standard way (recall $k$ is odd), and $\widetilde{\sigma}$ is the $\Sigma_{n}$-equivariant section 
of the map $e_{i}: e_{i}^{-1}\left(\operatorname{Conf}_{i}\left(S^{k}\right)\right) \rightarrow \operatorname{Conf}_{i}\left(S^{k}\right)$ obtained as the pullback in (3) of the assumed $\sigma$. Thus, for each $x \in S^{k}, c(x)$ is a multipath $\left\{c_{j}(x)\right\}_{j=0}^{i-1} \in\left(S^{k}\right)^{J_{i}}$, where each $c_{j}(x)$ is a path in $S^{k}$ starting at a point $s(x) \in S^{k}$ and ending at $z^{j} x$, for a continuous map $s: S^{k} \rightarrow S^{k}$. Note that the equivariance of $\tilde{\sigma}$ gives

$$
c_{j}(z x)=c_{j+1}(x)
$$

for all $x \in S^{k}$; here the value of $j$ is to be interpreted modulo $i$. Then the map $H: S^{k} \times[0,1] \rightarrow S^{k}$ defined by $H(x, t)=c_{0}(x)(t)$ is a homotopy starting at $s$ and ending at the identity. In particular, $s: S^{k} \rightarrow S^{k}$ has degree 1 . The contradiction comes by observing that the degree of $s$ would be divisible by $i$. Indeed, (16) gives

$$
s(z x)=c_{0}(z x)(0)=c_{1}(x)(0)=s(x),
$$

so that $s$ factors as $S^{k} \stackrel{\text { proj }}{\longrightarrow} L^{k}(i) \rightarrow S^{k}$, where $L^{k}(i)$ is the standard lens space $S^{k} /(\mathbb{Z} / i)$.

Corollary 5.5 The known equality $\mathrm{TC}_{2}^{S}\left(S^{k}\right)=2$ (valid for any integer $k>0$ ) extends to $\mathrm{TC}_{n}^{S}\left(S^{k}\right)=2(n-1)$ for $k=1$.

Remark 5.6 The first conclusion in Proposition 5.4 is partially extended by Karasev and Landweber's result in [19] asserting that $\mathfrak{g e n u s}\left(\varepsilon_{3}^{S^{k}}\right) \geq 1$ for $k$ not of the form $4 \cdot 3^{e}$ with $e \geq 0$. Note that the conjectural (13) would in fact sharpen the above estimate to an equality.

\section{References}

[1] I Basabe, J González, Y Rudyak, D Tamaki, Higher topological complexity and its symmetrization arXiv:1009.1851

[2] G E Bredon, Introduction to compact transformation groups, Pure and Applied Mathematics 46, Academic Press, New York (1972) MR0413144

[3] O Cornea, G Lupton, J Oprea, D Tanré, Lusternik-Schnirelmann category, Math. Surveys Monographs 103, Amer. Math. Soc. (2003) MR1990857

[4] A Dold, Lectures on algebraic topology, Classics in Mathematics, Springer, Berlin (1995) MR1335915

[5] M Farber, Topological complexity of motion planning, Discrete Comput. Geom. 29 (2003) 211-221 MR1957228

[6] M Farber, Instabilities of robot motion, Topology Appl. 140 (2004) 245-266 MR2074919 
[7] M Farber, Topology of robot motion planning, from: "Morse theoretic methods in nonlinear analysis and in symplectic topology", (P Biran, O Cornea, F Lalonde, editors), NATO Sci. Ser. II Math. Phys. Chem. 217, Springer, Dordrecht (2006) 185-230 MR2276952

[8] M Farber, Invitation to topological robotics, Zurich Lectures Adv. Math. 10, Eur. Math. Soc., Zürich (2008) MR2455573

[9] M Farber, M Grant, Symmetric motion planning, from: "Topology and robotics", (M Farber, R Ghrist, M Burger, D Koditschek, editors), Contemp. Math. 438, Amer. Math. Soc. (2007) 85-104 MR2359031

[10] M Farber, M Grant, Robot motion planning, weights of cohomology classes, and cohomology operations, Proc. Amer. Math. Soc. 136 (2008) 3339-3349 MR2407101

[11] M Farber, S Tabachnikov, S Yuzvinsky, Topological robotics: Motion planning in projective spaces, Int. Math. Res. Not. 2003 (2003) 1853-1870 MR1988783

[12] M Farber, S Yuzvinsky, Topological robotics: Subspace arrangements and collision free motion planning, from: "Geometry, topology, and mathematical physics", (V M Buchstaber, I M Krichever, editors), Amer. Math. Soc. Transl. Ser. 2 212, Amer. Math. Soc. (2004) 145-156 MR2070052

[13] E M Feichtner, G M Ziegler, The integral cohomology algebras of ordered configuration spaces of spheres, Doc. Math. 5 (2000) 115-139 MR1752611

[14] A M Gleason, Spaces with a compact Lie group of transformations, Proc. Amer. Math. Soc. 1 (1950) 35-43 MR0033830

[15] J González, P Landweber, Symmetric topological complexity of projective and lens spaces, Algebr. Geom. Topol. 9 (2009) 473-494 MR2491582

[16] N Iwase, M Sakai, Topological complexity is a fibrewise L-S category, Topology Appl. 157 (2010) 10-21 MR2556074

[17] J W Jaworowski, Extensions of $G$-maps and Euclidean $G$-retracts, Math. Z. 146 (1976) 143-148 MR0394550

[18] S Kallel, Symmetric products, duality and homological dimension of configuration spaces, from: "Groups, homotopy and configuration spaces", (N Iwase, T Kohno, R Levi, D Tamaki, J Wu, editors), Geom. Topol. Monogr. 13 (2008) 499-527 MR2508221

[19] R Karasev, P Landweber, Estimating the higher symmetric topological complexity of spheres, Algebr. Geom. Topol. 12 (2012) 75-94 MR2889546

[20] J-C Latombe, Robot motion planning, Kluwer Int. Series Engin. Comp. Sci. 124, Kluwer Academic, Boston (1991)

[21] S M LaValle, Planning algorithms, Cambridge Univ. Press (2006) MR2424564

[22] G Lupton, J Scherer, Topological complexity of $H$-spaces, Proc. Amer. Math. Soc. 141 (2013) 1827-1838 MR3020869 
[23] Y B Rudyak, On higher analogs of topological complexity, Topology Appl. 157 (2010) 916-920 MR2593704 Erratum in Topology Appl. (157) 20101118

[24] A S Švarc, The genus of a fiber space, Dokl. Akad. Nauk SSSR 119 (1958) 219-222 MR0102812 In Russian; translated in Amer. Math. Soc. Transl. 55 (1966) 49-140

IB,YBR: Department of Mathematics, University of Florida, 358 Little Hall, Gainesville, FL 32611-8105, USA

JG: Departamento de Matemáticas, CINVESTAV-IPN

A.P. 14-740, México City 07000, México

DT: Department of Mathematical Sciences, Shinshu University

Matsumoto 390-8621, Japan

iebasabe1@ufl.edu, jesus@math.cinvestav.mx, rudyak@ufl.edu, rivulus@shinshu-u.ac.jp

Received: 31 August 2013 